\title{
Effect of extinction trials on spontaneous blinking
}

EOWARD DEAUX AND KAY K, DEAUX

UNIVERSITY OF TEXAS ${ }^{1}$
Ss who received unpaired presentations of the CS and UCS following a series of paired presentations of the stimuli showed an immediate increase in spontaneous blink rate which was similar to but of longer duration than the increase shown by Ss for whom the UCS intensity was increased and the stimuli remained paired.

In an eyelid conditioning study recently conducted by one of the present writers (Spence \& Deaux, 1966), it was noted that the spontaneous blink rate (SBR) increased immediately after a stronger puff was introduced. Also observed was the apparent increase in SBR with no change in UCS intensity but with the introduction of unpaired presentations of the CS and UCS following the paired (acquisition) trials. In classical eyelid conditioning, intermixed CS- and UCS-alone presentations are frequently employed as a control condition for pseudoconditioning and are sometimes used in extinction, the UCS-alone presentations being assumed to maintain drive (D) at the level which obtained during the acquisition trials. If this observed increase in SBR is a real phenomenon, these uses of CS- and UCS-alone trials may not be appropriate, in that presentations of the separate stimuli result in increased $\mathrm{D}$. This argument assumes a positive relation between SBR and general drive level due to some sort of tension or stress. A similar suggestion has been made by Meyer (1953) and is supported by the evidence of several studies showing a relationship between SBR and a measure of tension (e.g., Ponder \& Kennedy, 1927; Telford \& Storlie, 1946).

The purpose of the present study was to determine whether the introduction of unpaired CS and UCS presentations produces such an increase in SBR. To accomplish this, three groups of Ss were run, two groups receiving paired (conditioning) trials throughout, one with a low and then a high puff strength and the other with the low puff strength maintained, and the third group changing from paired to unpaired (extinction) trials but with no change in puff strength. The expected outcome was that, whereas the control group which had no change in either stimulus presentations or UCS intensity would show small, nonsystematic fluctuations in number of blinks per minute, the Ss of the experimental groups who received either increased puff strength or change in type of trial would show a systematic increase in SBR following the change.

\section{Method}

The apparatus was identical to that employed by Spence \& Deaux (1966) with the addition of equipment to count all blinking responses made by S's right eyelid during the session. This consisted of an aluminum arm which was connected to the recording pen and broke the beam of a photo-contact relay with each response of at least $2 \mathrm{~mm}$ of pen deflection. A Hunter print-out counter recorded the blinks and printed the count once each minute during the session.

The CS consisted of a $500 \mathrm{cps}, 70 \mathrm{~dB}$ tone with a duration of $550 \mathrm{msec}$. presented over a continuous white noise background of $60 \mathrm{~dB}$ intensity. The UCS was a puff of compressed air, $50 \mathrm{msec}$. in duration, with an intensity of 0.5 or 2.0 psi depending on the experimental condition. The interstimulus interval was 500 msec., and both stimuli, when paired, terminated together. The $C R$ recording interval was from 100 to 500 msec, after CS onset.

The conditioning was imbedded in a game situation; $S$ was instructed to indicate on each trial which of two information lights was to follow a signal light, the CS and UCS being presented in this interval. The information lights followed a sequence of 3- and 4trial repeating patterns. The time between signal light offset and CS onset was $1.5 \mathrm{sec}$. On half the trials and 3.0 sec. on the other half, and other temporal relations were such that the mean CS-to-CS interval was $20 \mathrm{sec}$.

The Ss, 30 men and women students from an introductory psychology course, were randomly assigned to one of three groups, designated according to trial type and UCS intensity in the second phase as Group Paired-Low (PL), Group Paired-High (PH), and Group Unpaired-Low (UL). Preceding the start of Phase I, all Ss received three CS-alone and one UCS-alone presentations. In Phase I, all Ss were given $30 \mathrm{CS}-\mathrm{UCS}$ paired presentations, with a UCS intensity of $0.5 \mathrm{psi}$, randomly intermixed with 30 game-alone (GA) trials on which neither the CS nor the UCS was presented. In Phase II, Ss of Groups $\mathrm{PL}$ and $\mathrm{PH}$ received 30 CS-UCS paired presentations and $30 \mathrm{GA}$ trials as in Phase I, with a UCS intensity of 0.5 psi for Group PL and 2.0 psi for Group PH. Group UL received $30 \mathrm{CS}-$ alone and 30 UCS-alone presentations, randomly intermixed, during Phase $I I$ with a UCS intensity of 0.5 psi and no GA trials.

Thus Group PL received the same trial type and puff strength in both phases, Group PH had the puff 


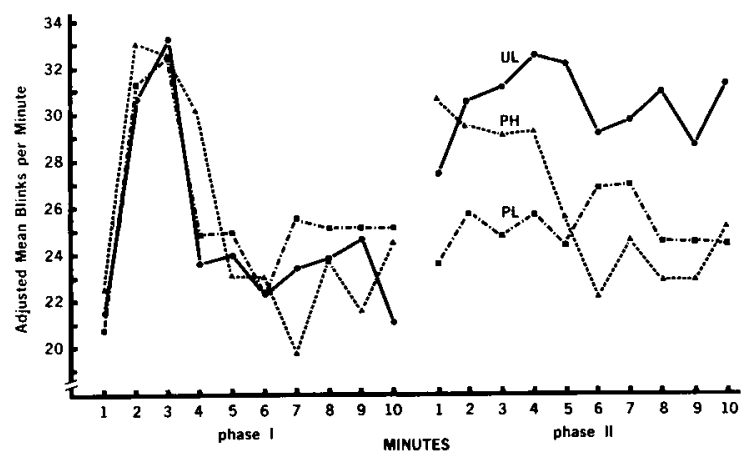

Fig. 1. Adjusted (see text) mean SBR per min. in each min. of both phases for Ss of Groups UL, PH, and PL.

strength increased and trial type unchanged, and Group UL had the puff strength unchanged and trial type altered from paired to unpaired presentations of the stimuli. Throughout both phases, all Ss received three UCS presentations per min.

\section{Results and Discussion}

The combined effects of large individual differences in SBR and relatively small Ns produced some differences between group blinking rates in Phase I, during which all Ss received the same treatment. To reduce these spurious differences in the graphical representation of the data, the groups' overall mean blink rates in Phase I were adjusted to be equal. Plotting according to this adjusted scale shows the Phase II changes in their proper magnitude. Figure 1 presents this graph. It can be seen that the introduction of the UCS at the start of Phase I produces a large increase in SBR, which, after about $4 \mathrm{~min}$., drops to a level only slightly above that of minute 1 . In Phase II, the Ss of Group PL continue to blink at a rather constant rate, as expected, while the increase in UCS intensity for $\mathrm{Ss}$ in Group $\mathrm{PH}$ produces an increase in SBR which is similar in magnitude and duration to that which occurred at the start of Phase I. Group UL shows a greater increase in SBR which does not subsequently return to the Phase I level. An analysis of covariance, which allowed for the influence in Phase II of the differences in the groups' Phase I rates, was conducted on the first 5 min. of Phase II and yielded an overall $F$ of $7.105(\mathrm{df}=2 / 26$, $\mathrm{p}<.01)$; specific comparisons with the covariance technique yielded an $\mathrm{F}$ of $1.014(\mathrm{df}=1 / 18, \mathrm{p}>.05)$ between Groups PH and UL and an F of 14.814 (df $=1 / 28$, $\mathrm{p}<.01$ ) between Group PL and Groups PH and UL combined. Thus, the amount of increase in the first half of Phase II is essentially the same for Groups PH and UL but significantly different from that of Group PL.

Whereas the increase in SBR in Group $\mathrm{PH}$ can be explained by increased irritation to $S$ 's eye, no such explanation can be applied as easily to the increased blinking of Group UL, since the latter group received the same low intensity UCS as Group PL. It might

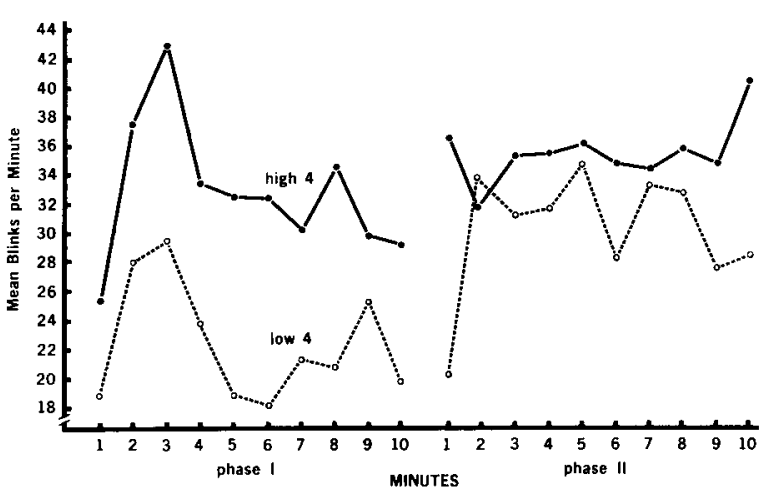

Fig. 2, Mean SBR per min. in each min. of both phases for the highest 4 vs. lowest 4 conditioned responders of Group UL.

be argued, however, that making a CR partially attenuates the noxious effects of the puff on CS-UCS paired trials and that, in extinction, removing the opportunity to thus decrease the puff's irritation is equivalent to increasing the puff strength. To check this possibility, Ss of Group UL were ranked according to number of CRs in Phase $I$, and the mean blink rates for the highest four and the lowest four conditioned responders were plotted for both phases. Figure 2 shows the curves for these two subgroups. The fact that the lowest four responders increased more than the highest four is exactly opposite to the argument's prediction and indicates that the cause of the increased SBR is not increased irritation. The positive relationship which is apparent in Fig. 2 between SBR and number of CRs in Phase I may be the result of two factors, operating singly or in combination: the increased probability of the occurrence of a response in the $\mathrm{CR}$ recording interval with increased SBR, and the increased facilitation of the response with increased motivational level as gauged by the higher SBR.

The predicted outcome that Groups $\mathrm{PH}$ and UL would both increase in SBR in Phase II was found. That Group UL did not return to its Phase I level, as did Group $\mathrm{PH}$, indicates a longer lasting effect in this group which may well be emotional.

\section{References}

Meyer, D. R. The interaction of simultaneous responses. Psychol. Bull., 1953, 50, 204-220.

Ponder, E., \& Kennedy, W. P. On the act of blinking. Quart. J. exp. Physiol., 1927, 18, 89-110.

Spence, K. W., \& Deaux, E. Conditioning (habit growth) in the absence of CRs. Psychon. Sci., 1966, 6, 61-62.

Telford, C. W., \& Storlie, A. The relation of respiration and reflex winking rates to muscular tension. $J$. exp. Psychol., 1946, 36, 512-517.

\section{Note}

1. This study was supported by NIMH fellowships no. $28,642-02$ and $30,398-02$ to the first and second author respectively. We thank Dr. Kenneth W. Spence and Dr. Janet T. Spence for their cooperation and assistance. 